\title{
Full-mouth Rehabilitation of a Ventriculoperitoneal Shunt-treated Hydrocephalic Pediatric Patient: A Case Report
}

\author{
Anish Poonia ${ }^{1}$, MM Dempsy Chengappa $^{2}$, Rajat Mitra ${ }^{3}$, Parul Jain ${ }^{4}$, Tarannum Ghavri ${ }^{5}$
}

\begin{abstract}
Hydrocephalus is characterized by accumulation of cerebrospinal fluid in the lateral ventricles of the brain, which results in progressive ventricular dilatation and an increased intracranial pressure. A ventriculoperitoneal shunt (VPS) is the most common treatment for hydrocephalus. Delayed development, behavioral disturbance, chronological changes in the eruption of their teeth, changes in the occlusion, greater accumulation of plaque, and increased caries prevalence are some of the clinical manifestations in these patients, which are encountered by a pediatric dentist. Behavioral disturbance in these patients makes sedation or general anesthesia the most feasible behavior management technique. During dental treatment of shunt-treated patients, care should be taken to avoid applying excessive force on the catheter. A referral to a neurosurgeon is recommended for patients with shunts undergoing invasive dental procedures for assessment of the need for prophylactic antibiotics. The present case report discusses full-mouth rehabilitation of a pediatric patient under general anesthesia who presented with severe early childhood caries and a medical history of ventriculoperitoneal shunt-treated hydrocephalus.
\end{abstract}

Keywords: General anesthesia, Hydrocephalus, Pediatric patient.

International Journal of Clinical Pediatric Dentistry (2020): 10.5005/jp-journals-10005-1744

\section{INTRODUCTION}

Hydrocephalus, the most common congenital malformation involving the central nervous system, with an incidence ranging from 0.4 to 0.8 cases per 1,000 births, ${ }^{1}$ is caused by an obstruction of fluid pathways or by excessive production of cerebrospinal fluid (CSF), which results in accumulation of CSF in the lateral ventricles of the brain. This leads to progressive ventricular dilatation and an increased intracranial pressure, which often damages the surrounding tissues. ${ }^{2}$

Cerebrospinal fluid shunting is the most common treatment for hydrocephalus. The ventriculoperitoneal shunt (VPS) used in the management of hydrocephalic patients incorporates a catheter that drains from the ventricles of the head to the abdominal regions. Ventriculoatrial shunts (VASs) and ventriculopleural shunts form the second and third choices for treatment. ${ }^{3}$

Despite the possibility of early diagnosis and surgical treatment, the prognosis for this disease is still not good and perinatal mortality is as high as $38 \% .{ }^{4}$ With growth, shunt-treated patients may exhibit facial asymmetry and malocclusion. Changes in the maturation of teeth (advanced during initial stages of puberty and delayed during the final stages) are also a common oral feature seen in this condition. $^{5-7}$

Children older than 2 or 3 years often present with headache, nausea, vomiting, irritability, lethargy, delayed development, behavioral disturbance, sunsetting eyes, visual complaints, bradycardia, hypertension, and irregular breathing patterns. As normal development is delayed, oral hygiene maintenance is often compromised in these child patients. Moreover, use of medications makes them more prone to dental caries. ${ }^{8}$

The American Academy of Pediatric Dentistry (AAPD) recognizes that routine dental care using nonpharmacologic behavior guidance techniques is not a viable approach for a selected population of patients, because of their need for extensive treatment, acute situational anxiety, uncooperative ageappropriate behavior, immature cognitive functioning, disabilities,
${ }^{1-5}$ Department of Pedodontics and Preventive Dentistry, Army Dental Centre, Research and Referral, New Delhi, India

Corresponding Author: Anish Poonia, Department of Pedodontics and Preventive Dentistry, Army Dental Centre, Research and Referral, New Delhi, India, Phone: +91 8968782990, e-mail: t.arora1908@gmail.com

How to cite this article: Poonia A, Chengappa MMD, Mitra R, et al. Full-mouth Rehabilitation of a Ventriculoperitoneal Shunt-treated Hydrocephalic Pediatric Patient: A Case Report. Int J Clin Pediatr Dent 2020;13(1):103-106.

Source of support: Nil

Conflict of interest: None

or medical conditions. Such patients would benefit from deep sedation or general anesthesia. ${ }^{9}$

The purpose of this paper is to report a case of full-mouth rehabilitation of a child patient who presented with severe early childhood caries and had a medical history of VPS-treated hydrocephalus.

\section{Case Description}

A 4.5-year-old male child reported with parents to the Department of Pedodontics and Preventive Dentistry with the chief complaint of pain and difficulty in eating food.

Medical history revealed that the child was born 20 days preterm. The child underwent endoscopic third ventriculostomy at 2 months of age and was operated for VPS at 5 months (Fig. 1). He was also operated for inguinal hernia at 2.5 years of age. On general examination, the child's measured height was $91 \mathrm{~cm}$ and weight was $11 \mathrm{~kg}$. He had an enlarged head circumference along with delayed developmental milestones. The catheter of VPS was palpable in the head and the cervical region. The child was on iron supplement medication in the first few years of life.

Dental history revealed that the child had undergone pulpal treatment for 61 and 62. Intraoral examination revealed dental 


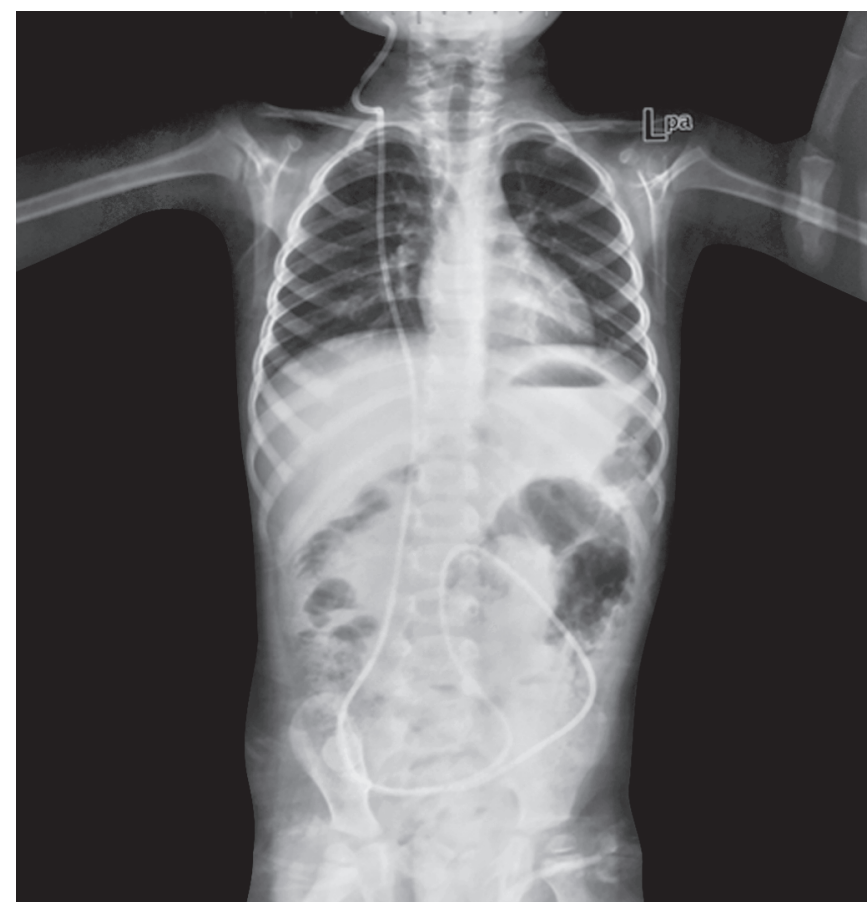

Fig. 1: Radiograph chest and abdomen showing a catheter in place

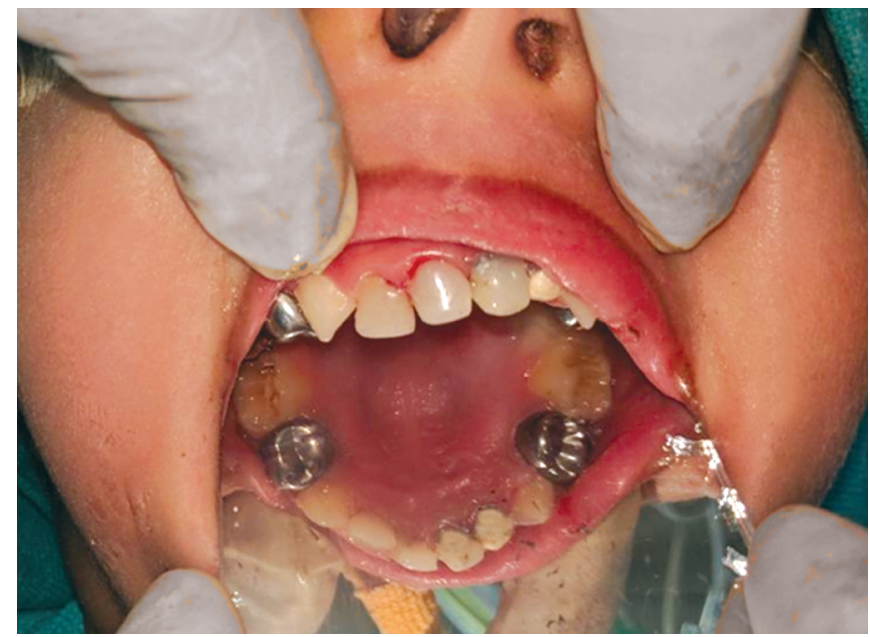

Fig. 3: Posttreatment maxillary occlusal view

caries involving 51, 52, 54, 64, 71, 72, 74, 75, 81, 82, 84, and 85 . Pulpectomy-treated 61 and 62 were present with absence of the crown structure (Fig. 2).

During the examination, he exhibited poor cooperation with sudden jerky movements. The child was rated as negative on the Frankl scale and was recognized as lacking cooperative ability. The panoramic radiograph could not be obtained because of sudden movements made by the child.

The patient was discussed in consultation with the neurosurgery department to evaluate the medical complications that can occur during invasive dental treatment and general anesthesia as well as the need for prophylactic antibiotics. The patient was cleared for undergoing full-mouth rehabilitation under general anesthesia with administration of prophylactic antibiotics.

Dental procedures carried out under general anesthesia included pulpectomies of $51,52,54,64,74,75,84$, and 85 ; strip crown

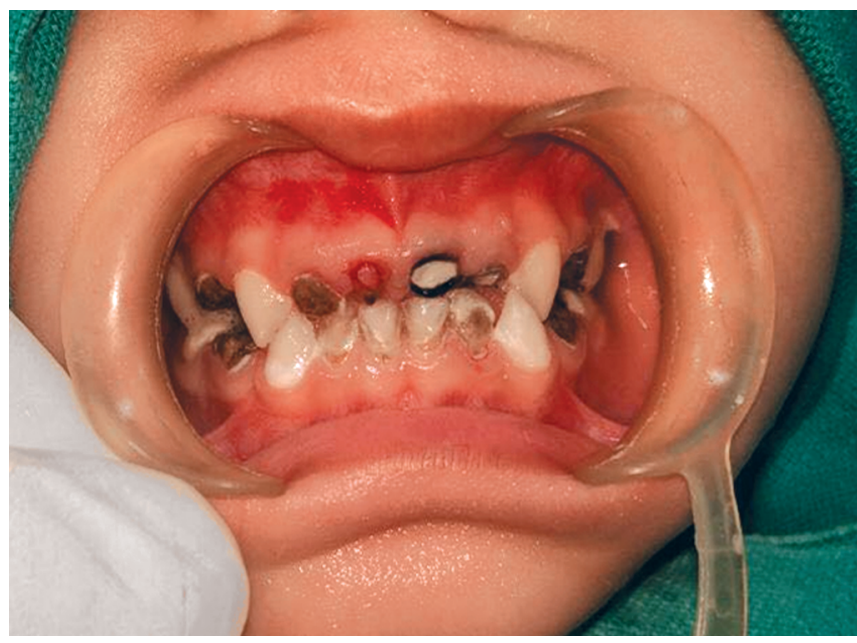

Fig. 2: Pretreatment intraoral view

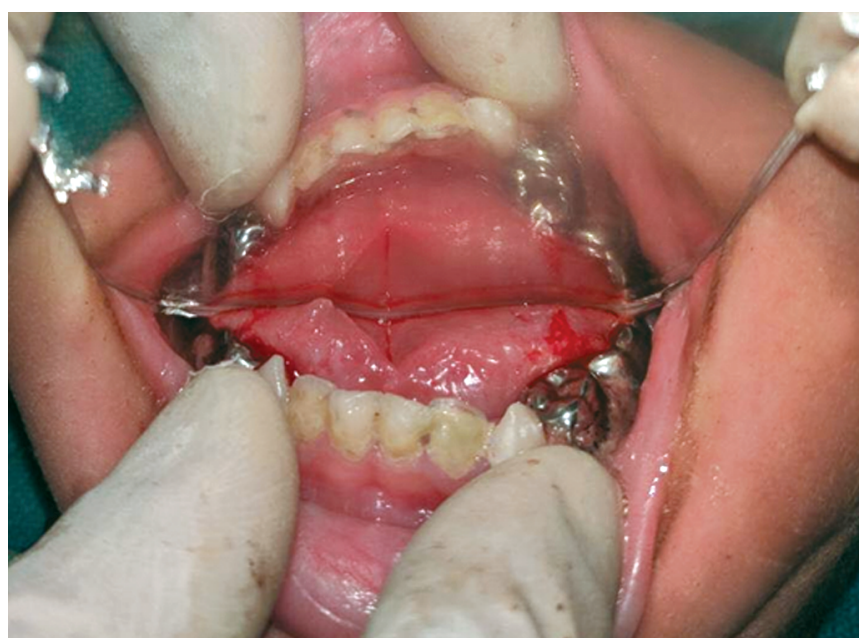

Fig. 4: Posttreatment mandibular occlusal view

restoration for 52; fiber post followed by strip crown restorations for 51 and 61 ; stainless steel crown restoration for $54,64,74,75,84$, and 85 ; and composite restoration for $71,72,81$, and 82 . Retention of fiber post in 62 was poor, so composite restoration was done for esthetic reasons (Figs 3 and 4). The postoperative restorations can be appreciated in the right and left lateral view in Figures 5 and 6 , respectively.

The patient was asymptomatic 1 month after surgery and was scheduled for the management of overall oral hygiene, development and eruption of permanent teeth, and structural change of the craniofacial region through periodic follow-up.

\section{Discussion}

With introduction of shunts, the mortality rate of hydrocephalic patients has decreased markedly along with a drastic improvement in the quality of life. This is characterized by a greater number of children with hydrocephalus seeking routine and emergency dental treatment making it important for pediatric dentists to have a thorough understanding of the condition and the associated dental considerations. ${ }^{10}$

In VPS-treated patients, the catheter is palpable subcutaneously in the head and the cervical region. This makes it necessary 


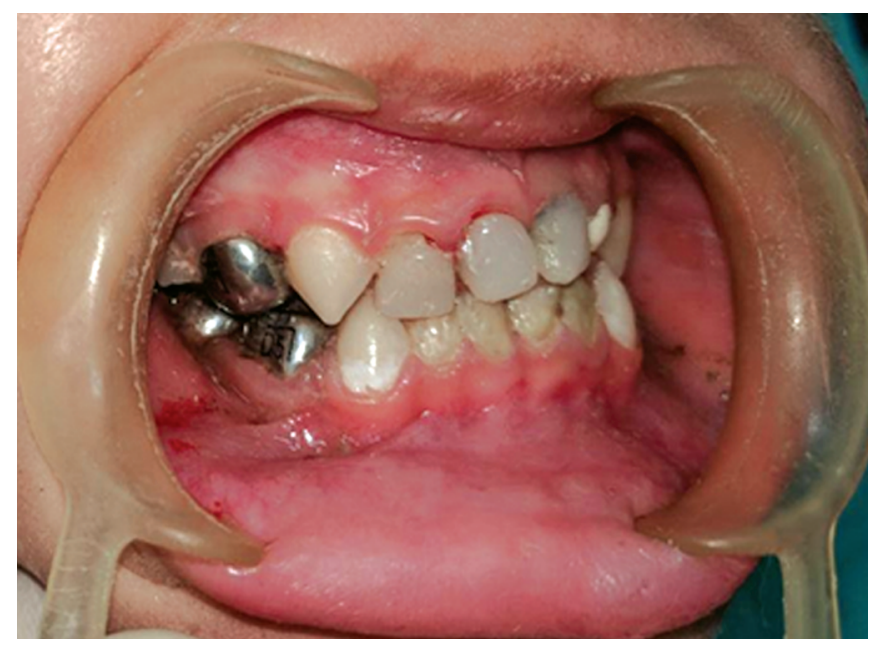

Fig. 5: Right frontal view

on part of a dental surgeon to exert caution, prevent exerting excessive force on the catheter during treatment procedures, and avoid complications of obstruction, fracture, and migration of the components. ${ }^{11}$ Although the exact dental situation was not described, de Carvalho et al. $^{12}$ reported a case of migration of a catheter in a 2-year-old child from the abdomen to the supraclavicular region caused by inadvertent mechanical forces to the shunt and vigorous movement of the patient's head. Pillows and cushions help to provide a more comfortable and adequate posture for the patient during dental treatment. ${ }^{11}$

A number of patients with intellectual and developmental disabilities in association with hydrocephalus experience fear and anxiety during dental care and treatment, making sedation or general anesthesia the most feasible behavior management techniques. ${ }^{13}$ With the exception of ketamine, which is associated with intracranial hypertension, to date, no sedative drug has been contraindicated in these patients. ${ }^{14}$

No consensus exists on the use of prophylactic antibiotics before dental procedures in shunt-treated patients. Scientific data on shunt infections following dental procedures are lacking. ${ }^{15}$ According to the most recent recommendations of the American Heart Association, ${ }^{16}$ antibiotic prophylaxis in invasive dental procedures is restricted only to patients with prosthetic cardiac valves, history of prior endocarditis, congenital heart disease, and cardiac transplant recipients who develop valvulopathy. In the present case, the medical opinion was that prophylactic antibiotics were required during dental treatment for the patient.

As regards restorative procedures, no modifications are required for patients with hydrocephalus. However, developmental disabilities seen in this children, difficulty on part of caregivers in providing adequate daily oral hygiene, ${ }^{8}$ the particular need for these patients to maintain good oral hygiene to avoid bacteremia and shunt infection, and possible health risks ${ }^{17}$ associated with pharmacological management approaches including their high costs make it important for these patients to be under a preventive protocol. Preventive measures being advocated include: avoiding frequent consumption of liquids and/or solid foods containing sugar, implementing oral hygiene measures no later than the time of eruption of the first primary tooth, providing professionally applied fluoride varnish treatments for children at risk for early childhood caries (ECC), and establishing a dental home within 6

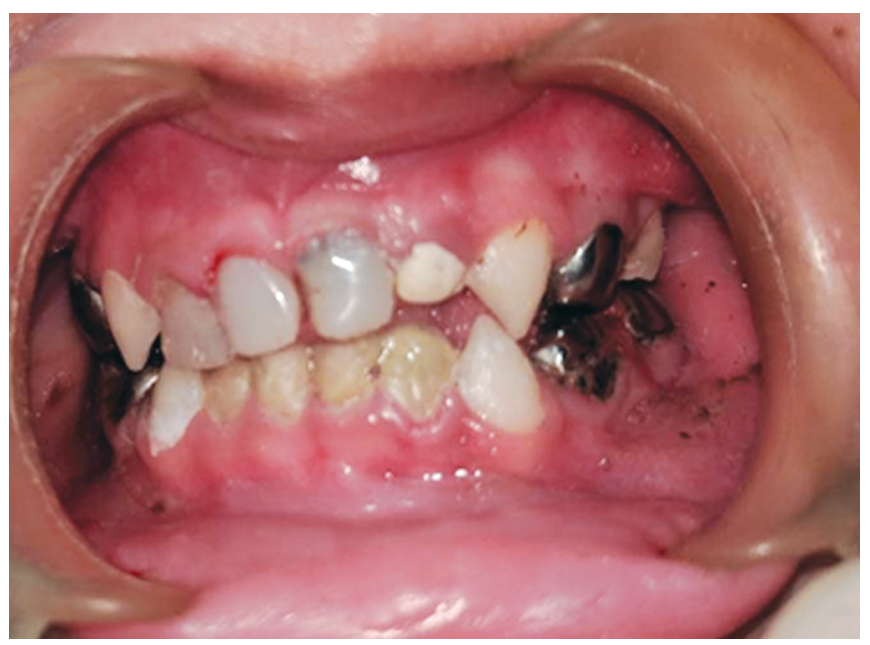

Fig. 6: Left frontal view

months of eruption of the first tooth and no later than 12 months of age, parental education, and awareness programs. ${ }^{18}$

\section{Conclusion}

A child with hydrocephalus managed with a VPS suffering from ECC will require extensive management to restore remaining dentition. The restoration of functional dentition is critical to improvement of the quality of life. These children need to be managed with care due to the presence of a shunt and to avoid various complications related to its obstruction, fracture, or migration. Preprocedure prophylaxis and postprocedure counseling of the parents and regular follow-up visits are the key to successful management of these children.

\section{References}

1. Schrander-Stumpel C, Fryns JP. Congenital hydrocephalus: nosology and guidelines for clinical approach and genetic counselling. Eur J Pediatr 1998;157(5):355-362. DOI: 10.1007/s004310050830.

2. Mori K, Shimada J, Kurisaka M, et al. Classification of hydrocephalus and outcome of treatment. Brain Dev 1995;17(5):338-348. DOI: 10.1016/0387-7604(95)00070-r.

3. Cartwright CC, Wallace DC. Nursing care of the pediatric neurosurgery patient. Springer-Verlag Berlin Heidelberg 2013. 444.

4. den Hollander NS, Vinkesteijn A, Schmitzvan Splunder $P$, et al. Prenatally diagnosed fetal ventriculomegaly; prognosis and outcome. Prenat Diagn 1998;18(6):557-566. DOI: 10.1002/(sici)10970223(199806)18:63.3.co;2-v.

5. Pirttiniemi PM, Huggare JA, Kantomaa TJ, et al. Craniofacial asymmetries in shunt-treated hydrocephalic children. Cleft Palate Craniofac J 1991;28(4):369-372. DOI: 10.1597/15451569_1991_028_0369_caisth_2.3.co_2.

6. Vilan Xavier AC, Pinto Silva LC, Oliveira P, et al. A review and dental management of persons with craniosynostosis anomalies. Spec Care Dentist 2008;28(3):96-100. DOI: 10.1111/j.1754-4505.2008.00019.x.

7. Pirttiniemi P, Poikela A, Huggare J, et al. Dental maturation in children with shunt-treated hydrocephalus. Cleft Palate Craniofac J 2004;41(6):651-654. DOI: 10.1597/03-018.1.

8. Lewis C, Robertson AS, Phelps S. Unmet dental care needs among children with special health care needs: implications for the medical home. Pediatrics 2005;116(3):e426-e431. DOI: 10.1542/peds.20050390.

9. American Academy of Pediatric Dentistry. Guideline on behavior guidance for the pediatric dental patient. Pediatr Dent 2005;27(7 Suppl):92-100. 
10. Massimi L, Paternoster G, Fasano T, et al. On the changing epidemiology of hydrocephalus. Childs Nerv Syst 2009;25(7):9971006. DOI: 10.1007/s00381-009-0844-4.

11. de Morais Gallarreta FW, Bernardotti FPL, de Freitas AC, et al. Characteristics of individuals with hydrocephalus and their dental care needs. Spec Care Dentist 2010;30(2):72-76. DOI: 10.1111/j.17544505.2009.00122.x.

12. de Carvalho RW, Pereira CU, Santos EA, et al. Retrograde migration of a ventriculoperitoneal shunt to the neck after dental treatment. J Dent Child (Chic) 2013;80(1):47-49.

13. Forsberg H, Quick-Nilsson I, Gustavson KH, et al. Dental health and dental care in severely mentally retarded children. Swed Dent J. 1985;9(1):15-28.

14. Dachs RJ, Innes GM. Intravenous ketamine sedation of pediatric patients in the emergency department. Ann Emerg Med 1997;29(1):146-150. DOI: 10.1016/s0196-0644(97) 70321-4.
15. Helpin ML, Rosenberg HM, Sayany Z, et al. Antibiotic prophylaxis in dental patients with ventriculo-peritoneal shunts: a pilot study. ASDC J Dent Child 1998;65(4):244-247.

16. Wilson W, TaubertKA, GewitzM, etal. Prevention of infectiveendocarditis: guidelines from the American Heart Association: a guideline from the American Heart Association Rheumatic Fever, Endocarditis, and Kawasaki Disease Committee, Council on Cardiovascular Disease in the Young, and the Council on Clinical Cardiology, Council on Cardiovascular Surgery and Anaesthesia, and the Quality of Care and Outcomes Research Interdisciplinary Working Group. Circulation 2007;116(15):1736-1754. DOI: 10.1161/CIRCULATIONAHA.106.183095.

17. Sinner B, Beck K, Engelhard K. General anesthetics and the developing brain: An overview. Anaesthesia 2014;69(9):1009-1022. DOI: 10.1111/ anae.12637.

18. American Academy of Pediatric Dentistry. Reference manual: Guideline on Perinatal and Infant Oral Health Care. Pediatr Dent 2016;38:16-17. 